8. M. Henriksen, Some remarks on a paper of Aronszajn and Panitchpakdi, Pacific J. Math. 7 (1957), 1619-1621.

9. J. R. Isbell and Z. Semadeni, Projection constants and spaces of continuous functions, Trans. Amer. Math. Soc. 107 (1963), 38-48.

10. J. Lindenstrauss, On the extension property for compact operators, Bull. Amer. Math. Soc. 68 (1962), 484-487.

11. - Extension of compact operators. II, Technical note no. 31, Jerusalem, June 1962; Trans. Amer. Math. Soc. (to appear).

12. - Extension of compact operators. III, Technical note no. 32, Jerusalem, July 1962; Trans. Amer. Math. Soc. (to appear).

13. L. Nachbin, A theorem of the Hahn-Banach type for linear transformations, Trans. Amer. Math. Soc. 68 (1950), 28-46.

14. A. Sobczyk, Projections of the space $m$ on its subspace $c_{0}$, Bull. Amer. Math. Soc. 47 (1941), 938-947.

YALE UNIVERSITY

\title{
ON THE STRUCTURE OF THE GREEN'S OPERATOR
}

\section{ROBERT CARROLL ${ }^{1}$}

1. Introduction. In the study of Cauchy problems of the form

$$
d u / d t+A u=f ; \quad u(\tau)=T
$$

(where for example: $t \rightarrow u(t) \in \mathcal{E}^{1}(H)$ on $(\tau, b] ; t \rightarrow u(t) \in \mathcal{E}^{0}(D(A))$ on $[\tau, b] ; H$ is a Hilbert space; $-A$ is a closed (unbounded) operator, infinitesimal generator of a strongly continuous semi-group; $\mathcal{E}^{k}(H)$ is the space of $k$-times continuously differentiable functions of $t$ with values in $H$; the domain of $A, D(A)$, has the graph topology; and $f, T$ are suitable), the solution takes the appearance

$$
u(t)=G(t, \tau) u(\tau)+\int_{\tau}^{t} G(t, \xi) f(\xi) d \xi .
$$

Formally the Green's operator $G(t, \xi)$ may be written $G(t, \xi)$ $=\exp [-A(t-\xi)]$ (for general results in this direction see for example $[1 ; 2 ; 3])$. In this article we propose to study representations related to (1.2) for solutions of general operational differential equations $S u=f$ (the operators need not be differential operators of course but therein lies the motivation, see $[4 ; 5]$; cf. also the papers $[3 ; 6 ; 7 ; 8$; 9; 10]).

2. Basic framework. Let $H$ be a Hilbert space and $\left(S_{0}, S_{0}^{\prime}\right)$ a formally adjoint pair of closed densely defined operators in the sense

Presented to the Society, April 19, 1963; received by the editors February 7, 1963.

1 Research supported in part by the Rutgers Research Council. 
of Browder [7]. Define $S_{1}=S_{0}^{*}$ (then $S_{0} \subset S_{1}$ ) and let $H_{0}=D\left(S_{0}\right)$, $H_{1}=D\left(S_{1}\right)$, where $H_{0}$ and $H_{1}$ have the graph topology. Then $H_{0} \subset H_{0} \subset H$ (algebraically and topologically) and following [10] we set $H_{1}=H_{0} \oplus B$ where $B$ is the so-called Cauchy space or space of abstract boundary conditions (see $[7 ; 9 ; 10]$ ). The symbol $\oplus$ denotes here an orthogonal direct sum (topological); when we wish to speak of a not necessarily orthogonal direct sum (topological) of two closed complementary subspaces $M_{1}$ and $M_{2}$ of a Hilbert space $M$ we will write $M=M_{1}+M_{2}$ (see here [11, p. 482]). It will be assumed throughout that $S_{0}$ is 1-1 with $S_{0}^{-1}$ continuous and that $S_{1}$ is onto $H$. (Such hypotheses are verified in many problems of interest; they imply (see [7]) that $S_{0}^{\prime}$ has a closed range $R\left(S_{0}^{\prime}\right)$ and that $\left(S_{0}, S_{0}^{\prime}\right)$ has a solvable realization operator $\tilde{S} ; R\left(S_{0}\right)$ is clearly closed also.) Now we will call any topological supplement of $H_{0}$ in $H_{1}$ a Cauchy space $\Gamma$ and write $H_{1}=H_{0}+\Gamma$ where in general $H_{0}$ and $\Gamma$ are not orthogonal. Clearly any such $\Gamma$ is isomorphic to $B$ (both are isomorphic to $H_{1} / H_{0}$ ). Then operators $\hat{S}$ such that $S_{0} \subset \hat{S} \subset S_{1}$ are characterized by linear subspaces $\hat{\Gamma}$ of $\Gamma$; that is, $\hat{H}=D(\hat{S})$ is the set $\left\{u_{1}: u_{1} \in H_{1} ; j u_{1} \in \hat{\Gamma} \subset \Gamma\right\}$ where $j: H_{1} \rightarrow \Gamma$ is the (open) projection determined by $H_{0}$ and $\Gamma$. Then $\hat{H}=H_{0}+\hat{\Gamma}$ and $\hat{H}$ would be given the graph topology. The following diagram will be useful in illustrating the subject (note ker $S_{1}$ is closed in $H$ or $H_{1}$ )

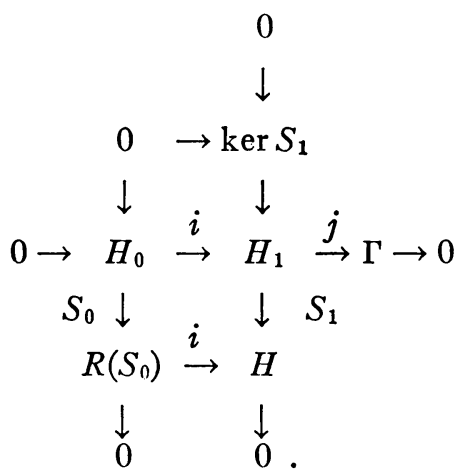

The horizontal and vertical sequences are exact (and split by the Banach theorem of homomorphisms). The continuous maps $i$ (injection), $S_{1}$, and $j$ (projection) may be thought of as morphisms in the category of Hilbert spaces. Note now that $H_{0}+\operatorname{ker} S_{1}$ is closed and hence a topological direct sum since if $u_{n}$ is Cauchy in $H_{0}+\operatorname{ker} S_{1}$ with $u_{n}=u_{0 n}+u_{1 n}$ then $S_{0} u_{0 n}$ converges which implies that $u_{0 n}$ converges. The diagram (2.1) may be further expanded as follows (cf. [7]), defining $\tilde{\Gamma}$ to be any topological supplement of $H_{0}+\operatorname{ker} S_{1}$ in $H_{1}$. 


$$
\begin{aligned}
H_{0}+ & \operatorname{ker} S_{1}+\tilde{\Gamma} \stackrel{j}{\rightarrow}\{0\}+\Gamma_{0}+\tilde{\Gamma} \\
& \downarrow S_{1} \\
R\left(S_{0}\right)+ & \{0\}+\tilde{H}
\end{aligned}
$$

where $\Gamma_{0}=j\left(\operatorname{ker} S_{1}\right), \tilde{H}=S_{1} \tilde{\Gamma}$, and in abuse of notation we identify $\tilde{\Gamma}$ and $j \tilde{\Gamma}$. It is clear that $\tilde{H}$ is closed since $S_{1}$ is open and an isomorphism on $\tilde{\Gamma}$; to see that $\tilde{H} \cap R\left(S_{0}\right)=\{0\}$, suppose the contrary. Thus if $h_{0} \in H_{0}, h \in \tilde{\Gamma}$, and $S_{0} h_{0}=S_{1} h$, it follows that $h_{0}-h \in \operatorname{ker} S_{1}$; since $h_{0}-h \in H_{1}-\operatorname{ker} S_{1}$ we must have $h_{0}-h=0$. Evidently $H=R\left(S_{0}\right)+\tilde{H}$.

We define the Green's operator to be the map $g:\left(j u_{1}, S_{1} u_{1}\right) \rightarrow u_{1}$ : $\Gamma \times H \rightarrow H_{1}$ which recovers $u_{1}$ from a knowledge of $j u_{1}$ and $S_{1} u_{1}$. It is seen from the diagrams that $g$ is well defined (if $j u_{1}=S_{1} u_{1}=0$ then $u_{1} \in$ ker $\left.S_{1} \cap H_{0}=\{0\}\right)$. Moreover suppose $j u_{1} \rightarrow 0$ in $\Gamma$ and $S_{1} u_{1} \rightarrow 0$ in $H$; then writing $u_{1}=u_{0}+u, u_{0} \in H_{0}, u \in \Gamma$, we have $u \rightarrow 0$ in $\Gamma$ and $S_{1} u+S_{0} u_{0} \rightarrow 0$ in $H$. Hence $u \rightarrow 0$ in $H, S_{1} u \rightarrow 0$ in $H$, and $S_{1} u+S_{0} u_{0} \rightarrow 0$ in $H$. This implies $S_{0} u_{0} \rightarrow 0$ in $H$ and therefore $u_{0} \rightarrow 0$ in $H$ by the continuity of $S_{0}^{-1}$. Thus finally $u_{1} \rightarrow 0$ in $H_{1}$ and we have

Proposition 1. The map $\mathrm{g}: \Gamma \times H \rightarrow H_{1}$ defined by $\mathcal{G}\left(j u_{1}, S_{1} u_{1}\right)=u_{1}$ is continuous.

It should be noted that $\mathcal{G}$ is not a bilinear map in the usual sense and is defined only on the set $G=\left\{\left(j u_{1}, S_{1} u_{1}\right)\right\} \subset \Gamma \times H$.

3. Decomposition of the Green's operator. By the preceding it follows that if $j u_{1}=0$ (i.e. $\left.u_{1} \in H_{0}\right)$ then $g\left(0, S_{1} u_{1}\right)$ defines a continuous map $\mathcal{G}_{2}: R\left(S_{0}\right) \rightarrow H_{1}$. Clearly on $R\left(S_{0}\right), \mathcal{G}_{2}$ may be written as $S_{0}^{-1}=\widetilde{S}^{-1}$ where $\tilde{S}$ is a solvable realization operator for $\left(S_{0}, S_{0}^{\prime}\right)$; hence $\mathcal{G}_{2}$ may be extended (as $\tilde{S}^{-1}$ ) to a continuous map $\mathcal{S}_{2}: H \rightarrow H_{0}+\tilde{\Gamma}$ (cf. [7]). On the other hand if $u_{1} \in \operatorname{ker} S_{1}$, then $\mathcal{G}\left(j u_{1}, 0\right)$ determines a continuous map $g_{1}: \Gamma_{0} \rightarrow H_{1}$ (the identity) which may be extended to a continuous map (the identity) $g_{1}: \Gamma_{0}+\tilde{\Gamma} \rightarrow H_{1}$. Then for $u_{1} \in H_{0}+\operatorname{ker} S_{1}$

$$
u_{1}=\mathcal{G}_{1}\left(j u_{1}\right)+\mathcal{G}_{2}\left(S_{1} u_{1}\right)
$$

whereas for $u_{1} \in \tilde{\Gamma}$ we must have

$$
u_{1}=\mathcal{G}_{1}\left(j u_{1}\right)=\mathcal{G}_{2}\left(S_{1} u_{1}\right) .
$$

Our interpretation of (1.2) is

$$
u_{1}=g_{2}\left(\rho S_{1} u_{1}\right)+G_{1}\left(j u_{1}\right),
$$

where $\rho: H \rightarrow R\left(S_{0}\right)$ is the projection, determined by $R\left(S_{0}\right)$ and $\tilde{H}$. Another formula for the solution similar to (3.3) is 


$$
u_{1}=g_{2}\left(S_{1} u_{1}\right)+\oint_{1}\left(\hat{\rho} j u_{1}\right),
$$

where $\hat{\rho}: \Gamma \rightarrow \Gamma_{0}$ is the projection, determined by $H_{0}$ and ker $S_{1}$. Note that the split $H_{0}+\operatorname{ker} S_{1}$ is predetermined; however there is still liberty in choosing $\tilde{\Gamma}$ and hence $\widetilde{H}$.

We recall now the notion of a kernel for an operator $T: \mathfrak{H C} \rightarrow \mathcal{H C}_{1}$ (see here for example $[12 ; 13 ; 14]$ ); we consider kernels in the sense of Aronszajn and will not attempt to treat here situations requiring the Schwartz kernel theorem (see [15]). Assuming $\mathfrak{H C}$ and $\mathfrak{K}_{1}$ are separable Hilbert spaces of equivalence classes of measurable functions over a regular measure space $(X, \mu)$ (see [12]), then $T$ has a kernel $T(y, \cdot)$ if: (1) for all $y \in X, T(y, \cdot) \in \mathcal{F}$; (2) the map $y \rightarrow T(y, \cdot): X \rightarrow \mathcal{F C}$ is measurable; (3) for all $h \in D(T),(T h)(y)=(h, T(y, \cdot))$ almost everywhere. If for example all functions in the range of a bounded operator $T$ are continuous then following Theorem 4 of [12] it is seen that $T$ has a kernel $T(y, \cdot)$. This will often prevail in applications (cf. [17]).

Suppose now that $g_{1}$ and $g_{2}$ have kernels $g_{1}(t, \cdot)$ and $g_{2}(t, \cdot) ; g_{1}$ and $\mathcal{G}_{2}$ are considered as operators in $\Gamma$ and $H$ respectively. Then for example (3.3) may be written (see [16] for extensions of (1.2))

$$
u_{1}=\left(\rho S_{1} u_{1}, g_{2}(t, \cdot)\right)_{H}+\left(j u_{1}, g_{1}(t, \cdot)\right)_{\Gamma} .
$$

We denote the adjoints of continuous maps $T$ by ${ }^{t} T$ and those of unbounded maps $T$ by $T^{*}$. Then from (3.5), since $g_{1} \in \Gamma$

$$
u_{1}=\left(u_{1},{ }^{t} S_{1}{ }^{t} \rho g_{2}(t, \cdot)+{ }^{t} j g_{1}(t, \cdot)\right)_{H_{1}} .
$$

The following exact sequences indicate how the maps work:

$$
\begin{aligned}
& 0 \rightarrow \tilde{H} \rightarrow H \stackrel{\rho}{\rightarrow} R\left(S_{0}\right) \rightarrow 0 ; \\
& 0 \rightarrow H \ominus R\left(S_{0}\right) \rightarrow H \stackrel{t^{\rho}}{\rightarrow} H \ominus \tilde{H} \rightarrow 0 ; \\
& 0 \rightarrow B_{0} \rightarrow B_{1} \stackrel{j}{\rightarrow} \Gamma \rightarrow 0 ; \\
& 0 \rightarrow H_{1} \ominus \Gamma \rightarrow H_{1} \stackrel{i j}{\rightarrow} H_{1} \ominus H_{0} \rightarrow 0 ; \\
& 0 \rightarrow \operatorname{ker} S_{1} \rightarrow H_{1} \stackrel{S_{1}}{\rightarrow} H \rightarrow 0 ; \\
& 0 \rightarrow H \stackrel{{ }^{t} S_{1}}{\rightarrow} H_{1} \ominus \operatorname{ker} S_{1} \rightarrow 0
\end{aligned}
$$

(note also ${ }^{t} S_{1}: R\left(S_{0}\right) \rightarrow H_{1} \ominus\left(\tilde{\Gamma}+\operatorname{ker} S_{1}\right)$ and ${ }^{t} S_{1}: \tilde{H} \rightarrow H_{1} \ominus\left(H_{0}+\operatorname{ker} S_{1}\right)$ ). It is seen that certain problems arise because of the fact that even if $H_{1}=\left(H_{0}+\operatorname{ker} S_{1}\right) \oplus \check{\Gamma}$ it is not true necessarily that $H=R\left(S_{0}\right) \oplus \check{H}$, where $\check{H}=S_{1} \check{\Gamma}$. For example if we choose $\check{H}$ first, orthogonal to 
$R\left(S_{0}\right)$, and define $\check{\Gamma}={ }^{t} S_{1} \check{H}$, then $\check{\Gamma}$ is orthogonal to $H_{0}+\operatorname{ker} S_{1}$; however then $S_{1} \check{\Gamma} \neq \check{H}$ in general.

Proposition 2. Assume $\mathcal{G}_{1}$ and $\mathrm{S}_{2}$ have kernels as above; then $H_{1}$ has a reproducing kernel given by

$$
h_{1}(t, \cdot)={ }^{t} S_{1}{ }^{t} \rho g_{2}(t, \cdot)+{ }^{t} j g_{1}(t, \cdot) .
$$

We may relate ${ }^{t} S_{1}$ to our original operators as follows. Assume $v \in H$ and ${ }^{t} S_{1} v=w$; then for all $u \in H_{1}$ we have $\left(S_{1} u, v\right)_{H}=(u, w)_{H_{1}}$. This means $\left(S_{1} u, v-S_{1} w\right)_{H}=(u, w)_{H}$. Therefore $v-S_{1} w \in D\left(S_{1}^{*}\right)$ and since $S_{1}^{*}=S_{0}^{\prime}$ it follows that $w=S_{0}^{\prime}\left(v-S_{1} w\right)$ (recall $H_{1}$ is dense in $H)$. Thus $w$ appears as a solution of the equation $\left(v-S_{1} w\right)=\left(S_{0}^{\prime}\right)^{-1} w$. We note that $g_{1}(t, \cdot)$ as defined is a reproducing kernel for $\Gamma$ and thus for $u_{1} \in \Gamma$ there results $u_{1}=\left(u_{1}, h_{1}\right)_{H_{1}}=\left(u_{1}, g_{1}\right)_{\Gamma}$. In general $g_{1}$ is the component of $h_{1}$ in $\Gamma$ when $H_{1}$ is written in the form $\Gamma \oplus\left(H_{1} \ominus \Gamma\right)$. It should be observed that $H_{0}$ orthogonal to ker $S_{1}$ in $H_{1}$ is impossible and this fact is closely connected with the development which we have given. A result similar to (3.7) can also be obtained using (3.4). By virtue of the above we may now write (3.7) in a form suitable for calculation.

$$
{ }^{t} \rho g_{2}=\left(\left(S_{0}^{\prime}\right)^{-1}+S_{1}\right)\left(h_{1}-{ }^{t} j g_{1}\right) .
$$

This formula will not however entirely determine $g_{2}$ in terms of $h_{1}$ and $g_{1}$; it defines $g_{2}$ up to a term in $H \ominus R\left(S_{0}\right)$. However, this is sufficient and we have

Proposition 3. The component of $g_{2}$ in $R\left(S_{0}\right)$ is determined by (3.8) if $h_{1}$ and $g_{1}$ are known. If therefore $\widetilde{H}$ is chosen orthogonal to $R\left(S_{0}\right)$ (with $\left.\tilde{\Gamma}=\widetilde{S}^{-1} \widetilde{H}\right)$, then $\mathcal{G}_{2}\left(\rho S_{1} u_{1}\right)$ is fully determined by (3.8).

On the other hand let $h_{1}$ be given; then $g_{1}$ is determined as the component of $h_{1}$ in $\Gamma$ when $H_{1}$ is decomposed as $H_{1}=\Gamma \oplus\left(H_{1} \ominus \Gamma\right)$. Thus if $J$ is the orthogonal projection $J: H_{1} \rightarrow \Gamma$ then $g_{1}=J h_{1}$. Define then the element ${ }^{t} \rho g_{2}={ }^{t} S_{1}^{-1}\left(h_{1}-{ }^{t} j g_{1}\right)$. This is well-defined since if $h_{1}=\tilde{h}_{1}+g_{1}$, $\tilde{h}_{1} \in H_{1} \ominus \Gamma, g_{1} \in \Gamma$, then ${ }^{t} j h_{1}={ }^{t} j g_{1}=\tilde{g}_{1} \in H_{1} \ominus H_{0}$ and since $t_{j}$ is a projection $h_{1}-\tilde{g}_{1} \in H_{1} \ominus \Gamma$; thus $h_{1}-{ }^{t} j g_{1} \in H_{1} \ominus \operatorname{ker} S_{1}$ with ${ }^{t} S_{1}^{-1}\left(h_{1}-\tilde{g}_{1}\right)$ well defined. Now since $h_{1}-\tilde{g}_{1} \in H_{1} \ominus \Gamma$ we have ${ }^{t} S_{1}^{-1}\left(h_{1}-{ }^{t} j g_{1}\right) \in R\left(S_{0}\right)$ and thus ${ }^{t} \rho g_{2} \in R\left(S_{0}\right)$. Assuming now $H=R\left(S_{0}\right) \oplus \tilde{H}$ with $\tilde{\Gamma}=\tilde{S}^{-1} \tilde{H}$, it follows that ${ }^{t} \rho g_{2}$ defines an element $g_{2}\left(={ }^{t} \rho g_{2}\right)$ in $R\left(S_{0}\right)$ with

$$
\begin{aligned}
\left(\rho S_{1} u_{1}, g_{2}\right)_{H} & =\left(S_{1} u_{1},{ }^{t} \rho g_{2}\right)=\left(S_{1} u_{1},{ }^{t} S_{1}^{-1}\left(h_{1}-{ }^{t} j g_{1}\right)\right) \\
& =\left(u_{1}, h_{1}-{ }^{t} j g_{1}\right)=u_{1}-\left(j u_{1}, g_{1}\right)=\mathcal{G}_{2}\left(\rho S_{1} u_{1}\right) .
\end{aligned}
$$

Hence $G_{2}$ has a kernel $g_{2}$ in $R\left(S_{0}\right)$ given by ${ }^{t} \rho^{-1}{ }^{t} S_{1}^{-1}\left(h_{1}-{ }^{t} j g_{1}\right)$. 
Proposition 4. Assume $H_{1}$ has a reproducing kernel $h_{1}$ and $H=R\left(S_{0}\right) \oplus \tilde{H}$. Then $\mathrm{S}_{2}$ has a kernel in $R\left(S_{0}\right)$ determined by (3.8).

Added in proof. The results of this paper are used in constructing abstract Green's operators in [16] for problems related to [5]. It is shown that $\hat{S}=\zeta^{*}$ (notations of [5]) and formulas such as (3.8) and (1.2) can be studied in more detail.

\section{BIBLIOGRAPHY}

1. T. Kato and H. Tanabe, On the abstract evolution equation, Osaka Math. J. 14 (1962), 107-133.

2. J. L. Lions, Les semi-groupes distributions, Portugal. Math. 19 (1960), 141-164.

3. - Equations différentielles-opérationnelles et problèmes aux limites, Springer, Berlin, 1961.

4. R. Carroll, Quelques problèmes d'opérateurs reliês, C. R. Acad. Sci. Paris 255 (1962), 1371-1373.

5. - Problems in linked operators. I, Math. Ann. 151 (1963), 272-282.

6. J. L. Lions and E. Magenes, Problemi ai limiti non omogenei, Ann. Scuola Norm. Sup. Pisa 14 (1960), 269-308; 15 (1961), 39-101; 15 (1961), 311-326; 16 (1962), 1-44; Problèmes aux limites non homogènes, Ann. Inst. Fourier (Grenoble) 11 (1961), 137-178.

7. F. Browder, Functional analysis and partial differential equations, Math. Ann. 138 (1959), 55-79; 145 (1962), 6-226.

8. - On the spectral theory of elliptic differential operators, Math. Ann. 142 (1961), 22-130.

9. R. S. Phillips, Dissipative operators and hyperbolic systems of partial differential equations, Trans. Amer. Math. Soc. 90 (1959), 193-254.

10. H. O. Cordes, On maximal first order partial differential operators, Amer. J. Math. 82 (1960), 63-91.

11. N. Dunford and J. Schwartz, Linear operators, Interscience, New York, 1958.

12. E. Nelson, Kernel functions and eigenfunction expansions, Duke Math. J. 25 (1958), 15-27.

13. N. Aronszajn, Green's functions and reproducing kernels, Proc. Sympos. on Spectral Theory and Differential Problems, Stillwater, Oklahoma, 1955.

14. H. Meschkowski, Hilbertsche Räume mit Kernfunktion, Springer, Berlin, 1962.

15. L. Schwartz, Thérie des distributions a valeurs vectorielles, Ann. Inst. Fourier (Grenoble) 7 (1957), 1-141; 8 (1958), 1-209.

16. R. Carroll, Problems in linked operators. II (to appear).

17. N. Aronszajn and K. Smith, Characterization of positive reducing kernels. Applications to Green's functions, Amer. J. Math. 79 (1957), 611-622.

Rutgers, The State University 